\title{
Incomplete convolutions in production and inventory models
}

\author{
G.J. van Houtum, W.H.M. Zijm \\ University of Twente, Faculty of Mechanical Engineering, Production and Operations Management Group, P.O. Box 217, 7500 AE Enschede, The Netherlands \\ (Phone: +31 53 4893621, Fax: +31 53 4893471, e-mail: w.h.m.zijm@wb.utwente.nl)
}

Received: 15 April 1996 / Accepted: 8 January 1997

\begin{abstract}
In this paper, we study incomplete convolutions of continuous distribution functions, as they appear in the analysis of (multi-stage) production and inventory systems. Three example systems are discussed where these incomplete convolutions naturally arise. We derive explicit, nonrecursive formulae for these convolutions, for the relevant case in which the underlying distributions are (mixtures of) Erlang distributions with the same scale parameter. Numerical results for one example system, the multi-stage serial inventory system, are presented to show the effectiveness of these formulae.

Zusammenfassung. In der vorliegenden Arbeit werden sog. unvollständige Faltungen stetiger Verteilungsfunktionen untersucht, wie sie bei der Analyse (mehrstufiger) Produktionsund Lagerhaltungssysteme auftreten können. Es werden beispielhaft drei Systeme betrachtet, in denen solche unvollständigen Faltungen typischer Weise vorkommen. Für den wichtigen Fall, daß die zugrundeliegenden Wahrscheinlichkeitsverteilungen (Mischungen von) Erlang-Verteilungen mit identischem Skalenparameter sind, werden explizite nichtrekursive Formeln für diese Faltungen abgeleitet. Schließlich werden für ein Anwendungsbeispiel, bestehend aus dem Fall eines mehrstufigen, seriellen Lagerhaltungssystems, numerische Ergebnisse vorgestelit, die die Effektivität der angegebenen Formeln zeigen.
\end{abstract}

Key words: Production systems, inventory systems, (incomplete) convolutions

Schlüsselwörter: Produktionssysteme, Lagerhaltungssysteme, (unvollständige) Faltungen

\section{Introduction}

Let $G_{1}$ and $G_{2}$ be continuous distribution functions on $[0, \infty)$ with $G_{1}(0)=G_{2}(0)=0$ and let $a$ be a real-valued, nonnegative variable. Then the distribution function $G_{1}^{a}$ is defined by

$G_{1}^{a}(x):= \begin{cases}G_{1}(x+a) & \text { if } x \geq 0 \\ 0 & \text { if } x<0,\end{cases}$ and the distribution function $G_{1}^{a} * G_{2}$, where $*$ denotes the usual convolution operator for the distribution functions of two independent random variables, is called an incomplete convolution because of its alternative representation:

$\left(G_{1}^{a} * G_{2}\right)(x)=\int_{0}^{x} G_{1}(x+a-u) \mathrm{d} G_{2}(u), \quad x \geq 0$.

Let $X_{1}$ and $X_{2}$ be independent random variables which are distributed according to $G_{1}$ and $G_{2}$, respectively. Then the function $G_{1}^{a}$ is the distribution function of the random variable $\left(X_{1}-a\right)^{+}$, where $x^{+}=\max \{0, x\}$ for all $x \in \boldsymbol{R}$, and the incomplete convolution $G_{1}^{a} * G_{2}$ is the distribution function of $\left(X_{1}-a\right)^{+}+X_{2}$. Random variables of the latter type naturally arise in the analysis of (multi-stage) production and inventory systems, and therefore it is worth to devote a separate study to their distribution functions.

The function $G_{1}^{a} * G_{2}$ is called a 2 -fold incomplete convolution. In general, we are dealing with $r$-fold incomplete convolutions, with $r \geq 1$ :

$$
\begin{aligned}
\hat{G}_{r}(x):= & \left(\left(\ldots\left(G_{1}^{a_{1}} * G_{2}\right)^{a_{2}} * \ldots * G_{r-1}\right)^{a_{r-1}} * G_{r}\right)(x), \\
& x \geq 0,
\end{aligned}
$$

where $G_{1}, \ldots, G_{r}$ are continuous distribution functions on $[0, \infty)$ with $G_{1}(0)=\ldots=G_{r}(0)=0$ and $a_{1}, \ldots, a_{r-1}$ are real-valued, nonnegative variables. The function $\hat{G}_{r}$ alternatively follows from the following recursive definition: $\hat{G}_{1}(x):=G_{1}(x), x \geq 0$, and for $s=1, \ldots, r-1$, $\hat{G}_{s+1}(x):=\left(\hat{G}_{s}^{a_{s}} * G_{s+1}\right)(x), \quad x \geq 0$.

Let $X_{1}, \ldots, X_{r}$ be independent random variables which are distributed according to the distribution functions $G_{1}, \ldots$, $G_{r}$. Then $\hat{G}_{1}$ is the distribution function of $\hat{X}_{1}:=X_{1}$, and for $s=1, \ldots, r-1, \hat{G}_{s+1}$ is the distribution of $\hat{X}_{s+1}:=$ $\left(\hat{X}_{s}-a_{s}\right)^{+}+X_{s+1}$. So, $\hat{G}_{r}$ is the distribution function of $\hat{X}_{r}$; it is easily verified that (cf. (1))

$$
\begin{aligned}
\hat{X}_{r}= & \left(\ldots\left(\left(X_{1}-a_{1}\right)^{+}+X_{2}-a_{2}\right)^{+}+\ldots\right. \\
& \left.+X_{r-1}-a_{r-1}\right)^{+}+X_{r} .
\end{aligned}
$$

Note that this random variable can be interpreted as the sojourn time in a $D|G| 1$ queueing system where each customer has its own deterministic interarrival time and random service time (this system is also known as a service system with 


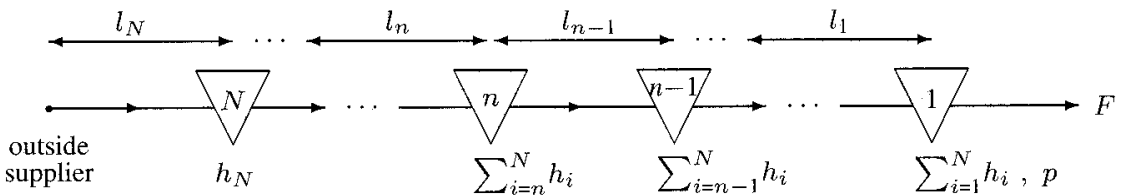

Fig. 1. The $N$-stage serial inventory system appointment times, see e.g. Gerchak et al. [4]); see also the remarks at the end of Subsect. 2.2.

This paper is completely devoted to incomplete convolutions and it is organized as follows. In Sect. 2, we describe three examples of inventory and production systems where incomplete convolutions play an important role in the analysis and in the representation of the minimal cost policy. These examples are: a multi-stage serial inventory system (cf. Clark and Scarf [2]), a capacitated single-stage inventory system (cf. Federgruen and Zipkin [3]) and a multistage serial production system (cf. Yano [14] and Gong et al. [5]). Next, in Sect. 3, we derive explicit, non-recursive formulae for $r$-fold incomplete convolutions for the special case in which all underlying distributions $G_{1}, \ldots, G_{r}$ are Erlang distributions with the same scale parameter. For $r$ fold incomplete convolutions with general $r \geq 1$, this is the only case (up to our knowledge) for which such formulae have been derived. After that, in Sect. 4, we show that these formulae also can be used in the relevant case where all underlying distributions are mixtures of Erlang distributions which all have the same scale parameter. The obtained formulae will be exploited to efficiently compute the optimal (base stock) policies and the corresponding costs for several instances of the multi-stage serial inventory system. Finally, some concluding remarks are presented in Sect. 5.

\section{Example systems}

In each of the three subsections of this section, we describe an example of a production/inventory system in which incomplete convolutions naturally arise in the analysis. We give a brief description of the example systems itself, show how the incomplete convolutions arise, and list the main results. As one will see, one example concerns a (capacitated) single-stage system, while the other two examples concern (uncapacitated) multi-stage systems with a simple, serial structure. It is noted, however, that the incomplete convolutions also arise in the analysis of both assembly systems (which are equivalent to serial systems under certain circumstances) and distribution systems; see e.g. [13].

\subsection{A multi-stage serial inventory system}

Consider the $N$-stage serial inventory system $(N \geq 2)$ (cf. Clark and Scarf [2]), which has been displayed in Figure 1. Materials, parts or products can be ordered from any installation and are then shipped to the next downstream installation. The system is subject to periodic review. Any order for materials placed at the beginning of a period $t$ with destination installation $n$ arrives at that installation at the beginning of period $t+l_{n}$ ( $l_{n} \in N ; l_{1}$ is also allowed to be equal to 0 ), at least if sufficient materials are available at the upstream installation. The outside supplier preceding installation $N$ can always deliver. Outside demand is experienced solely at installation 1 at the end of the chain. In each period, the demand is nonnegative and continuously distributed with distribution function $F$. We assume that $F(0)=0$, i.e. $F$ has no positive probability mass in 0 . Demands in different periods are assumed to be independent. The distribution of the $l$-period cumulative demand is given by the $l$-fold convolution of $F$, denoted by $F_{l}$.

Materials at installation $n$ or in transit from installation $n$ to $n-1$ are subject to inventory holding costs, per item per period, equal to $h_{N}+\ldots+h_{n}(\geq 0)$, for $n=N, \ldots, 2$. Materials at installation 1 are subject to inventory holding costs equal to $h_{N}+\ldots+h_{1}(\geq 0)$. In principle, it is allowed to take $h_{n} \leq 0$ for some $n$. Any excess demand is backlogged. In case of a shortage at installation 1, a penalty $p(>0)$ per item per period is incurred (no penalty costs are incurred in case of a shortage at upstream installations, i.e. only real customer delivery problems are penalized). Both the inventory holding and the penalty costs are incurred at the end of a period. No fixed and/or linear ordering costs are assumed.

The inventory holding and penalty costs can also be expressed based on the echelon stocks of the different installations. The echelon stock of a given installation denotes all stock at that installation plus all materials in transit to or on hand at any installation downstream minus a possible back$\log$ at the most downstream installation. The corresponding chain under consideration is called the echelon, and its number is according to its highest installation. An echelon stock may be negative, indicating that the backlog is larger than the total inventory in that echelon. Now, let $v_{n}$ denote the stock of echelon $n$ at the end of a period (note that the above definitions imply $v_{n} \geq v_{n-1}$ for $n=2, \ldots, N$ ). It is easily verified (cf. $[12,15])$ that the total inventory holding and penalty costs in that period are equal to

$\begin{cases}\sum_{n=1}^{N} h_{n} v_{n} & \text { if } v_{1} \geq 0 \\ \sum_{n=1}^{N} h_{n} v_{n}-(p+H) v_{1} & \text { if } v_{1}<0,\end{cases}$

where $H=\sum_{n=1}^{N} h_{n}$. The costs $h_{n} v_{n}$ are called the costs attached to echelon $n, n=2, \ldots, N$, and the remaining costs are called the costs attached to echelon 1. Note that the terms $h_{n} v_{n}$ always appear, independently of the sign of $v_{n}$.

We are interested in determining an ordering policy that minimizes the average costs in the infinite horizon case. Since it is known that the class of base stock policies always contains an overall optimal policy (cf. [2]), we can restrict ourselves to this class.

A base stock policy operates on the basis of the echelon inventory positions of the different installations. The echelon inventory position of an installation is defined as its echelon stock plus all materials which are in transit to that installation. If it is assumed that an installation never orders more than what is available at the next upstream installation, then 
this definition of echelon inventory position is equivalent to defining the echelon inventory position as the echelon stock plus all materials which are on order. A base stock policy is denoted by a tuple $\left(y_{1}, \ldots, y_{N}\right)$, where $y_{n}(\in \boldsymbol{R})$ denotes the desired order-up-to level for the echelon inventory position of installation $n$. Under a base stock policy $\left(y_{1}, \ldots, y_{N}\right)$, at the beginning of each period, the echelon inventory position of installation $N$ is increased to $y_{N}$, and for each installation $n=N-1, \ldots, 1$, the echelon inventory position is increased to the minimum of $y_{n}$ and the actual echelon stock of the next upstream installation, i.e. of installation $n+1$ (the start up phenomena, occurring in case the initial echelon inventory positions are larger than the desired levels, define a transient phase of the system and hence may be ignored).

Next, we show that the average costs $g\left(y_{1}, \ldots, y_{N}\right)$ of a given base stock policy $\left(y_{1}, \ldots, y_{N}\right)$ can be expressed in terms of incomplete convolutions.

Consider the actual levels to which the echelon inventory positions of the installations $1, \ldots, N$ are increased under the given base stock policy $\left(y_{1}, \ldots, y_{N}\right)$. The variables $y_{1}, \ldots, y_{N}$ denote the desired order-up-to levels. Note that the level $y_{n}$ for an installation $n$ never will be reached if one of the higher installations has a smaller desired order-up-to level. Hence, it is better to analyze how the actual levels deviate from the adjusted desired order-up-to levels defined by

$\tilde{y}_{n}:=\min \left\{y_{n}, \ldots, y_{N}\right\} \quad$ for $n=1, \ldots, N$.

Note that $\tilde{y}_{1} \leq \tilde{y}_{2} \leq \ldots \leq \tilde{y}_{N}$.

The difference between $\tilde{y}_{n}$ and the actual level to which the echelon inventory position of an installation $n$ is increased at the beginning of an arbitrary period is called the shortfall and it is denoted by the random variable $\tilde{Y}_{n}$. Since the outside supplier can always deliver, the shortfall $\tilde{Y}_{N}$ of the highest installation $N$ is equal to 0 by definition. For $n<N$, the shortfall $\tilde{Y}_{n}$ can be determined from $\tilde{Y}_{n+1}$ as follows. Consider an arbitrary period $t$. Then $\tilde{y}_{n+1}-\tilde{Y}_{n+1}$ is the actual level to which the echelon inventory position of installation $n+1$ is increased at the beginning of that period $t$, and thus the echelon stock of installation $n+1$ at the beginning of period $t+l_{n+1}$ is given by $\tilde{y}_{n+1}-\tilde{Y}_{n+1}-U_{l_{n+1}}$, where the random variable $U_{l_{n+1}}$ represents the total demand in the periods $t$ up to and including $t+l_{n+1}-1$ (so, $U_{l_{n+1}}$ has distribution function $F_{l_{n+1}}$ ). As a result, at the beginning of period $t+l_{n+1}$ (which also represents an arbitrary period), the echelon inventory position of installation $n$ can be increased to

$$
\begin{aligned}
& \min \left\{\tilde{y}_{n}, \tilde{y}_{n+1}-\tilde{Y}_{n+1}-U_{l_{n+1}}\right\}= \\
& \tilde{y}_{n}-\left(\tilde{Y}_{n+1}+U_{l_{n+1}}-\left(\tilde{y}_{n+1}-\tilde{y}_{n}\right)\right)^{+},
\end{aligned}
$$

and hence its shortfall is given by

$$
\tilde{Y}_{n}=\left(\tilde{Y}_{n+1}+U_{l_{n+1}}-\left(\tilde{y}_{n+1}-\tilde{y}_{n}\right)\right)^{+} \text {. }
$$

Exploiting this recurrence relation shows that the distribution function of $\tilde{Y}_{n}, n=1, \ldots, N$, is given by (note that this distribution function only depends on $\left.y_{n}, \ldots, y_{N}\right)$ :

$$
\begin{aligned}
\tilde{F}_{n, N}^{\left[y_{n}, \ldots, y_{N}\right]}(x):= & \left(\left(\ldots\left(F_{l_{N}}^{b_{N}} * F_{l_{N-1}}\right)^{b_{N-1}} * \ldots\right.\right. \\
& \left.\left.* F_{l_{n+2}}\right)^{b_{n+2}} * F_{l_{n+1}}\right)^{b_{n+1}}(x), x \in \boldsymbol{R}
\end{aligned}
$$

(for $n=N$, read $\hat{F}_{N, N}^{\left[y_{N}\right]}(x):=I(x)$, defined by $I(x)=1$ for $x \geq 0$ and $I(x)=0$ for $x<0$ ), where $b_{i}:=\tilde{y}_{i}-\tilde{y}_{i-1}$ for $i=2, \ldots, N$.

We now can obtain the echelon stocks of the different installations at the end of an arbitrary period. For each installation $n, n=1, \ldots, N$, the echelon inventory position at the beginning of an arbitrary period $t$ is increased to $\tilde{y}_{n}-\tilde{Y}_{n}$, which results in an echelon stock at the end of period $t+l_{n}$ equal to $\tilde{y}_{n}-\left(\tilde{Y}_{n}+U_{l_{n}+1}\right)$, where $U_{l_{n}+1}$ represents the total demand in the periods $t$ up to and including $t+l_{n}$. This demand $U_{l_{n}+1}$ is independent of $\tilde{Y}_{n}$ and hence the distribution function of $\tilde{Y}_{n}+U_{l_{n}+1}$ is given by the $(N-n+1)$-fold incomplete convolution

$$
\begin{aligned}
F_{n, N}^{\left[y_{n}, \ldots, y_{N}\right]}(x):= & \left(\left(\ldots\left(F_{l_{N}}^{b_{N}} * F_{l_{N-1}}\right)^{b_{N-1}} * \ldots\right.\right. \\
& \left.\left.* F_{l_{n+1}}\right)^{b_{n+1}} * F_{l_{n}+1}\right)(x), x \in \boldsymbol{R}
\end{aligned}
$$

(for $n=N$, read $F_{N N}^{\left[y_{N}\right]}(x):=F_{l_{N}+1}(x), x \in \boldsymbol{R}$ ). By combining this result with (2), we obtain the following Newsboytype formula for the average costs $g\left(y_{1}, \ldots, y_{N}\right)$ (cf. Van Houtum and Zijm [12]):

$$
\begin{aligned}
g\left(y_{1}, \ldots, y_{N}\right)= & \sum_{n=1}^{N} h_{n} \int_{0}^{\infty}\left(\tilde{y}_{n}-u\right) \mathrm{d} F_{n, N}^{\left[y_{n}, \ldots, y_{N}\right]}(u) \\
& +(p+H) \int_{\tilde{y}_{1}}^{\infty}\left(u-\tilde{y}_{1}\right) \mathrm{d} F_{1, N}^{\left[y_{1}, \ldots, y_{N}\right]}(u) .
\end{aligned}
$$

The description above shows the natural appearance of incomplete convolutions when analyzing the average costs of a base stock policy. The incomplete convolutions also show up in the characterization of the optimal base stock policy. This is seen in the following brief list of main results for the $N$-stage serial inventory system (these results have been gathered from $[2,6,12]$; for an extensive overview of these and additional results, see [13]):

(i) The class of base stock policies is optimal, i.e. it contains an overall optimal policy.

(ii) An optimal base stock policy $\left(S_{1}, \ldots, S_{N}\right)$ is obtained as follows: for $n=1, \ldots, N$, choose the order-up-to level $S_{n}$ for the echelon inventory position of installation $n$ such that

$F_{1, n}^{\left[S_{1}, \ldots, S_{n-1}, S_{n}\right]}\left(\tilde{S}_{1}^{n}\right)=\frac{p+\sum_{i=n+1}^{N} h_{i}}{p+H}$

where $\tilde{S}_{1}^{n}=\min \left\{S_{1}, \ldots, S_{n}\right\}$ and for all $y_{1}, \ldots, y_{n}$, the incomplete convolution $F_{1, n}^{\left[y_{1}, \ldots, y_{n}\right]}$ is defined by

$$
\begin{aligned}
F_{1, n}^{\left[y_{1}, \ldots, y_{n}\right]}(x):= & \left(\left(\ldots\left(F_{l_{n}}^{b_{n}} * F_{l_{n-1}}\right)^{b_{n-1}} * \ldots\right.\right. \\
& \left.\left.* F_{l_{2}}\right)^{b_{2}} * F_{l_{1}+1}\right)(x), x \in \boldsymbol{R}
\end{aligned}
$$

(for $\left.n=1, \operatorname{read} F_{1,1}^{\left[y_{1}\right]}(x):=F_{l_{1}+1}(x), x \in \boldsymbol{R}\right)$, with

$$
\begin{array}{ll}
b_{i}:=\tilde{y}_{i}-\tilde{y}_{i-1} & \text { for } i=2, \ldots, n, \\
\tilde{y}_{i}:=\min \left\{y_{i}, \ldots, y_{n}\right\} & \text { for } i=1, \ldots, n .
\end{array}
$$

(iii) The average costs $g\left(S_{1}, \ldots, S_{N}\right)$ of the optimal policy $\left(S_{1}, \ldots, S_{N}\right)$ are obtained by the formula stated in (3).

Result (ii) shows that for the determination of an optimal base stock policy, it is not required to solve an $N$ dimensional minimization problem, but it suffices to solve 


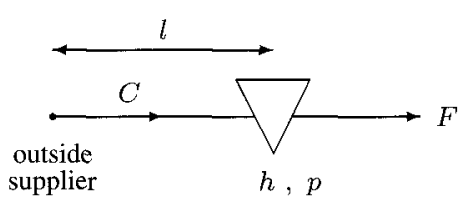

Fig. 2. The capacitated single-stage inventory system

$N$ one-dimensional problems. An optimal base stock policy $\left(S_{1}, \ldots, S_{N}\right)$ is obtained by first choosing $\left(S_{1}, \ldots, S_{N}\right)=$ $(\infty, \ldots, \infty)$ and next for $n=1, \ldots, N$ decreasing $S_{n}$ such that the probability for a nonnegative stock at installation 1 at the end of an arbitrary period (this probability is given by $F_{1, n}^{\left[S_{1}, \ldots, S_{n-1}, S_{n}\right]}\left(\tilde{S}_{1}^{n}\right)$ ) becomes equal to $\left(p+\sum_{i=n+1}^{N} h_{i}\right) /(p+H)$ (note that this ratio is non-increasing for increasing values of $n$ if all inventory holding cost parameters $h_{n}$ are nonnegative). Here, in each step, the value of $S_{n}$ can be tuned by a bisection procedure (the probability $F_{1, n}^{\left[S_{1}, \ldots, S_{n-1}, y\right]}\left(\tilde{S}_{1}\right)$ with $\tilde{S}_{1}=\min \left\{S_{1}, \ldots, S_{n-1}, y\right\}$ is non-decreasing as a function of $y$, as easily follows from the definition of incomplete convolutions given in Sect. 1). It is noted that an optimal base stock policy $\left(S_{1}, \ldots, S_{N}\right)$ does not necessarily have nondecreasing order-up-to levels $S_{1} \leq \ldots \leq S_{N}$

The results listed above show that we can compute an optimal base stock policy for the $N$-stage serial inventory system if we are able to evaluate incomplete convolutions $\hat{G}_{r}$ (cf. (1)) where each of the underlying distributions is a convolution of one generic distribution $F$ (the demand distribution). The corresponding average costs can be determined if we are able to evaluate integrals of the type $\int_{a_{r}}^{\infty}\left(u-a_{r}\right) \mathrm{d} \hat{G}_{r}(u)$ (choose $a_{r}=0$ for the integrals in the first term of formula (3) and choose $a_{r}=\tilde{y}_{1}$ for the integral in the second term; note that for $a_{r}=0$ the integral gives the expectation corresponding to the distribution $\hat{G}_{r}$ ).

\subsection{A capacitated single-stage inventory system}

Consider the capacitated single-stage inventory system with leadtime $l(l \in N \cup\{0\})$, demand distribution $F$ (a continuous distribution function on $[0, \infty)$ with $F(0)=0$ ), fixed capacity $C$ (larger than the mean demand per period), inventory holding costs $h(\geq 0)$ and penalty costs $p(>0)$ (cf. Federgruen and Zipkin [3]); see Figure 2. For this system, the same assumptions hold as for the multi-stage serial inventory system studied in the previous subsection. An additional assumption is that in each period the amount that can be ordered at (or delivered by) the outside supplier is bounded by a fixed size $C$.

Again we are interested in determining an ordering policy that minimizes the average costs over an infinite horizon, and again the class of base stock policies is known to contain such an optimal policy (cf. [3]).

In this case a base stock policy is denoted by a single variable $y \in \boldsymbol{R}$. The corresponding average costs $g(y)$ can be determined by studying the shortfall process. Let $\tilde{Y}$ denote the shortfall, i.e. the difference between the desired orderup-to level $y$ and the actual order-up-to level of the (echelon) inventory position of the single installation, at the beginning of an arbitrary period. Then $\tilde{Y}=\lim _{t \rightarrow \infty} \tilde{Y}_{t}$, where $\tilde{Y}_{t}, t \geq$ 0 , denotes the shortfall at the beginning of period $t_{0}+t$ while it is given that the shortfall at the beginning of some period $t_{0}$ is equal to 0 . So, $\tilde{Y}_{0}=0$ and for $t \geq 0$,

$\tilde{Y}_{t+1}=\left(\tilde{Y}_{t}+U_{t}-C\right)^{+}$,

where $U_{t}$ represents the demand in period $t_{0}+t$. For the distribution functions $\tilde{F}_{t}$ of the random variables $\tilde{Y}_{t}$, it holds that $\tilde{F}_{0}(x)=I(x)$, with $I(x)=0$ for all $x<0$ and $I(x)=1$ for all $x \geq 0$, and for each $t \geq 0, \tilde{F}_{t+1}(x)=\left(\tilde{F}_{t} * F\right)^{C}(x)$, $x \geq 0$. Note that the shortfall process $\left\{\tilde{Y}_{t}\right\}_{t \geq 0}$ and its distributions do not depend on $y$.

For each $t \geq 0$, it holds that the (echelon) stock at the end of period $t_{0}+t+l$ is equal to $y-\left(\tilde{Y}_{t}+U_{t, t+l}\right)$, where $U_{t, t+l}$ represents the total demand in the periods $t_{0}+t$ up to and including $t_{0}+t+l$. The distribution function of $\tilde{Y}_{t}+U_{t, t+l}$ is given by the $(l+1)$-fold incomplete convolution

$\hat{F}_{t}(x)=\left(\tilde{F}_{t} * F_{l+1}\right)(x), \quad x \geq 0$,

and the distribution function $\hat{F}$ of the difference between $y$ and the stock of the single installation at the end of an arbitrary period follows from $\hat{F}(x)=\lim _{t \rightarrow \infty} \hat{F}_{t}(x), x \geq 0$. By the latter distribution, we find the average costs $g(y)$ of the base stock policy $y$ :

$g(y)=h \int_{0}^{\infty}(y-u) \mathrm{d} \hat{F}(u)+(p+h) \int_{y}^{\infty}(u-y) \mathrm{d} \hat{F}(u)$.

From the derivative of this convex function, it follows that an optimal base stock policy $S$ is characterized by (cf. Tayur [9])

$\hat{F}(S)=\frac{p}{p+h}$.

The analysis above shows that an optimal base stock policy $S$ and the corresponding average costs $g(S)$ can be computed if we are able to evaluate incomplete convolutions $\hat{G}_{r}$ (cf. (1)) and integrals of the type $\int_{a_{r}}^{\infty}\left(u-a_{r}\right) \mathrm{d} \hat{G}_{r}(u)$ for the case in which the underlying distributions $G_{1}, \ldots, G_{r-1}$ are equal to a generic distribution $F$ (the demand distribution), the underlying distribution $G_{r}$ is equal to an $(l+1)$-fold convolution of $F$ and the variables $a_{1}, \ldots, a_{r-1}$ are identical (and equal to the fixed capacity $C$ ).

The recurrence relation for the shortfall process $\left\{\tilde{Y}_{t}\right\}_{t \geq 0}$, as stated in (4), shows that this process is identical to the waiting time process in a $D|G| 1$ queue with deterministic interarrival times $C$ and service times which are distributed according to $F$ (cf. $[12,9]$ ). In fact, $\tilde{Y}_{t}$ represents the waiting time of the $t$-th customer and the distribution functions of the waiting and response time of this customer are given by $\tilde{F}_{t}$ and $\tilde{F}_{t} * F$, respectively. Further, the distribution functions of the waiting and response time of an arbitrary customer are given by $\tilde{F}$ and $\tilde{F} * F$, respectively. This shows that the incomplete convolutions $\hat{G}_{r}$ as defined by (1) are also relevant for the $D|G| 1$ queue.

\subsection{A multi-stage serial production system}

In this subsection, we consider an $\mathrm{N}$-stage serial production system $(N \geq 2)$ (cf. Gong et al. [5]; see also Yano [14], 


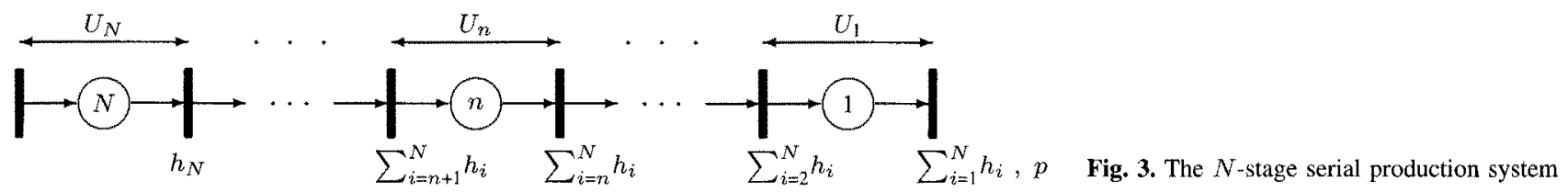

who studied the system with $N=2$ stages). This system is displayed in Figure 3. The system consists of $N$ consecutive stages, which are numbered from $N, \ldots, 1$, and it produces products (or batches of products) on order. For each stage $n$, the processing time is given by a generic random variable $U_{n}$ with continuous distribution function $F_{n}$ with $F_{n}(0)=0$; the processing times at different stages are assumed to be independent. At the end of each stage $n>1$, a product may wait in a dispatch area before it enters the next stage. At the end of stage 1, a product may have to wait before it is delivered to its customer. A product is delivered at its due date, or, if not possible, immediately after its completion. A penalty $p(>0)$ is incurred per unit of time that a product is delivered after its due date. Further, for each $n>1$, holding $\operatorname{costs} \sum_{i=n}^{N} h_{i}(\geq 0)$ are incurred per unit of time that a product waits in the dispatch area at the end of stage $n$ and per unit of time that a product is being processed at stage $n-1$. Holding costs $H=\sum_{i=1}^{N} h_{i}$ are incurred per unit of time between the completion of a product at stage 1 and the time instant at which the product is delivered to its customer.

The production system operates under a dispatch policy $\left(z_{1}, \ldots, z_{N}\right)$, where each $z_{n}(\in \boldsymbol{R})$ denotes the planned leadtime for the subsystem consisting of the stages $n, \ldots, 1$ (so, each $z_{n}$ defines an echelon leadtime). If $d$ is the due date of an order, then for each $n=N, \ldots, 1$, the corresponding product is dispatched at stage $n$ at time instant $d-z_{n}$, or as soon as possible if the previous stage is not completed at that time instant (here, for $n=N$, the previous stage is constituted by the order acceptance stage).

The objective is to find a dispatch policy that minimizes the average holding and penalty costs for each order.

As recognized by Gong et al. [5] (see also [15]), the problem to find the optimal dispatch policy is equivalent to finding the optimal base stock policy for an $N$-stage serial inventory system. Hence, we can copy the main results that we have stated in Subsect. 2.1. For the $N$-stage serial production system, the main results read as follows:

(i) An optimal dispatch policy $\left(T_{1}, \ldots, T_{N}\right)$ is obtained as follows: for $n=1, \ldots, N$, choose the planned leadtime $T_{n}$ such that

$$
F_{1, n}^{\left[T_{1}, \ldots, T_{n-1}, T_{n}\right]}\left(\tilde{T}_{1}^{n}\right)=\frac{p+\sum_{i=n+1}^{N} h_{i}}{p+H},
$$

where $\tilde{T}_{1}^{n}=\min \left\{T_{1}, \ldots, T_{n}\right\}$ and for all $z_{1}, \ldots, z_{n}$, the incomplete convolution $F_{1, n}^{\left[z_{1}, \ldots, z_{n}\right]}$ is defined by

$$
\begin{aligned}
F_{1, n}^{\left[z_{1}, \ldots, z_{n}\right]}(x):= & \left(\left(\ldots\left(F_{n}^{b_{n}} * F_{n-1}\right)^{b_{n_{1}-1}} * \ldots\right.\right. \\
& \left.\left.* F_{2}\right)^{b_{2}} * F_{1}\right)(x), x \in \boldsymbol{R}
\end{aligned}
$$

(for $n=1$, read $F_{1,1}^{\left[z_{1}\right]}(x):=F_{1}(x), x \in \boldsymbol{R}$ ), with

$$
\begin{array}{ll}
b_{i}:=\tilde{z}_{i}-\tilde{z}_{i-1} & \text { for } i=2, \ldots, n, \\
\tilde{z}_{i}:=\min \left\{z_{i}, \ldots, z_{n}\right\} & \text { for } i=1, \ldots, n .
\end{array}
$$

(ii) The average costs $g\left(T_{1}, \ldots, T_{N}\right)$ of the optimal dispatch policy $\left(T_{1}, \ldots, T_{N}\right)$ are obtained from the following formula for the average costs $g\left(z_{1}, \ldots, z_{N}\right)$ of an arbitrary dispatch policy $\left(z_{1}, \ldots, z_{N}\right)$ :

$$
\begin{aligned}
g\left(z_{1}, \ldots, z_{N}\right)= & \sum_{n=1}^{N} h_{n} \int_{0}^{\infty}\left(\tilde{z}_{n}-u\right) \mathrm{d} F_{n, N}^{\left[z_{n}, \ldots, z_{N}\right]}(u) \\
& +(p+H) \int_{\tilde{z}_{1}}^{\infty}\left(u-\tilde{z}_{1}\right) \mathrm{d} F_{1, N}^{\left[z_{1}, \ldots, z_{N}\right]}(u),
\end{aligned}
$$

where for each $n=1, \ldots, N$,

$$
\begin{aligned}
F_{n, N}^{\left[z_{n}, \ldots, z_{N}\right]}(x):= & \left(\left(\ldots\left(F_{N}^{b_{N}} * F_{N-1}\right)^{b_{N-1}} * \ldots\right.\right. \\
& \left.\left.* F_{n+1}\right)^{b_{n+1}} * F_{n}\right)(x), x \in \boldsymbol{R}
\end{aligned}
$$

(for $n=N$, read $F_{N, N}^{\left[z_{N}\right]}(x):=F_{N}(x), x \in \boldsymbol{R}$ ), and

$$
\begin{array}{ll}
b_{i}:=\tilde{z}_{i}-\tilde{z}_{i-1} & \text { for } i=2, \ldots, N, \\
\tilde{z}_{i}:=\min \left\{z_{i}, \ldots, z_{N}\right\} & \text { for } i=1, \ldots, N .
\end{array}
$$

The average costs $g\left(T_{1}, \ldots, T_{N}\right)$ denote the holding and penalty costs for orders that are accepted at least $T_{N}$ time units before its due date (and, for each of these orders the probability that it is delivered in time is equal to $\left.F_{1, N}^{\left[T_{1}, \ldots, T_{N}\right]}\left(\tilde{T}_{1}^{N}\right)=p /(p+H)\right)$. The expected costs for an order that is accepted only $z<T_{N}$ time units before its due date, are given by $g\left(T_{1}, \ldots, T_{N-1}, z\right)$.

As we see, to compute an optimal dispatch policy and the expected holding and penalty costs of an order, we must be able to evaluate incomplete convolutions $\hat{G}_{r}$ (cf. (1)) and integrals of the type $\int_{a_{r}}^{\infty}\left(u-a_{r}\right) \mathrm{d} \hat{G}_{r}(u)$, where no additional properties are given for the underlying distributions $G_{1}, \ldots, G_{r}$.

\section{Explicit formulae of incomplete convolutions of Erlang distributions with the same scale parameter}

Consider the $r$-fold incomplete convolution $\hat{G}_{r}$ as given in (1). In this section, we derive explicit, non-recursive formulae for the functions $\hat{G}_{r}, r \geq 1$, for the special case where the underlying distributions $G_{1}, G_{2}, \ldots$ are Erlang distributions with the same scale parameter. These formulae in fact express the functions $\hat{G}_{r}$ as finite sums of simple terms (see Theorem 1), and hence by using them one can avoid numerical integration (and/or discretization) to evaluate the functions $\hat{G}_{r}$. Note that these formulae are relevant for the multi-stage, serial inventory system and the capacitated, single-stage inventory system, where we have to deal with underlying distributions which are equal to either convolutions of the demand distribution $F$ or $F$ itself. Hence, the underlying distributions are equal to Erlang distributions with the same scale parameter, if the demand distribution $F$ itself is equal to an Erlang distribution. The formulae that 
we derive also can be exploited if the demand distribution $F$ is equal to a mixture of Erlang distributions with the same scale parameter, as we shall discuss in the next section.

The main part of this section is devoted to the derivation of the explicit formulae for the functions $\hat{G}_{r}$ itself. At the end of this section, we show that these formulae can be exploited to obtain similar formulae for the integrals of the type $\int_{a_{r}}^{\infty}\left(u-a_{r}\right) \mathrm{d} \hat{G}_{r}(u)$ (see Theorem 2).

Let $E_{k, \lambda}$ denote the Erlang distribution consisting of $k \in$ $N$ phases and with scale parameter $\lambda$, i.e.

$E_{k, \lambda}(x)=1-\sum_{i=0}^{k-1} \frac{(\lambda x)^{i}}{i !} e^{-\lambda x}, \quad x \geq 0$.

Throughout this section, we assume that $\lambda$ is a real-valued, positive variable, $k_{1}, k_{2}, \ldots$ are positive integers and $a_{1}$, $a_{2}, \ldots$ are real-valued, nonnegative variables, and we focus on the incomplete convolutions $\hat{H}_{r}, r \geq 1$, which we define by: $\hat{H}_{1}(x):=E_{k_{1}, \lambda}(x), x \geq 0$, and for $r \geq 1$,

$$
\hat{H}_{r+1}(x):=\left(\hat{H}_{r}^{a_{r}} * E_{k_{r+1}, \lambda}\right)(x), \quad x \geq 0 .
$$

So, for all $r \geq 1$,

$$
\begin{aligned}
\hat{H}_{r}(x)= & \left(\left(\ldots\left(\left(E_{k_{1}, \lambda}\right)^{a_{1}} * E_{k_{2}, \lambda}\right)^{a_{2}} * \ldots\right.\right. \\
& \left.\left.* E_{k_{r-1}, \lambda}\right)^{a_{r-1}} * E_{k_{r}, \lambda}\right)(x), x \geq 0
\end{aligned}
$$

(compare to (1)).

The one-fold incomplete convolution $\hat{H}_{1}$ is identical to $E_{k_{1}, \lambda}$ and hence its formula is given by (5) with $k=k_{1}$. Of more interest is the two-fold incomplete convolution $\hat{H}_{2}$. For this function, we find

$$
\begin{aligned}
\hat{H}_{2}(x)= & \left(\left(E_{k_{1}, \lambda}\right)^{a_{1}} * E_{k_{2}, \lambda}\right)(x) \\
= & \int_{0}^{x} E_{k_{1}, \lambda}\left(x+a_{1}-u\right) \mathrm{d} E_{k_{2}, \lambda}(u) \\
= & \int_{0}^{x}\left(1-\sum_{i=0}^{k_{1}-1} \frac{\left(\lambda\left(x+a_{1}-u\right)\right)^{i}}{i !} e^{-\lambda\left(x+a_{1}-u\right)}\right) \\
& \times \lambda^{k_{2}} \frac{u^{k_{2}-1}}{\left(k_{2}-1\right) !} e^{-\lambda u} \mathrm{~d} u \\
= & E_{k_{2}, \lambda}(x)-\sum_{i=0}^{k_{1}-1} \lambda^{i+k_{2}} e^{-\lambda\left(x+a_{1}\right)} \\
& \times \int_{0}^{x} \frac{\left(x+a_{1}-u\right)^{i}}{i !} \frac{u^{k_{2}-1}}{\left(k_{2}-1\right) !} \mathrm{d} u, x \geq 0 .
\end{aligned}
$$

For the integral in this formula, we obtain a simple formula, that is given by the following lemma; here, $N_{0}:=N \cup\{0\}$.

Lemma 1. For all $i_{1}, i_{2} \in \boldsymbol{N}_{0}$ and $x_{1}, x_{2} \in \boldsymbol{R}$ with $x_{1}, x_{2} \geq$ 0 ,

$$
\int_{0}^{x_{2}} \frac{\left(x_{2}+x_{1}-u\right)^{i_{1}}}{i_{1} !} \frac{u^{i_{2}}}{i_{2} !} \mathrm{d} u=\sum_{j=0}^{i_{1}} \frac{x_{1}^{i_{1}-j}}{\left(i_{1}-j\right) !} \frac{x_{2}^{j+i_{2}+1}}{\left(j+i_{2}+1\right) !} .
$$

Proof. When $x_{1}=0$, the result to be proved reads as follows: for all $i_{1}, i_{2} \in N_{0}$ and $x_{2} \in R, x_{2} \geq 0$,

$$
\int_{0}^{x_{2}} \frac{\left(x_{2}-u\right)^{i_{1}}}{i_{1} !} \frac{u^{i_{2}}}{i_{2} !} \mathrm{d} u=\frac{x_{2}^{i_{1}+i_{2}+1}}{\left(i_{1}+i_{2}+1\right) !} .
$$

This result for the special case $x_{1}=0$ is proved by repeated partial integration, and it readily leads to the formula for the case with general $x_{1} \geq 0$ :

$$
\begin{aligned}
& \int_{0}^{x_{2}} \frac{\left(x_{2}+x_{1}-u\right)^{i_{1}}}{i_{1} !} \frac{u^{i_{2}}}{i_{2} !} \mathrm{d} u \\
& =\int_{0}^{x_{2}} \frac{1}{i_{1} !}\left(\sum_{j=0}^{i_{1}}\left(\begin{array}{c}
i_{1} \\
j
\end{array}\right) x_{1}^{i_{1}-j}\left(x_{2}-u\right)^{j}\right) \frac{u^{i_{2}}}{i_{2} !} \mathrm{d} u \\
& =\sum_{j=0}^{i_{1}} \frac{x_{1}^{i_{1}-j}}{\left(i_{1}-j\right) !} \int_{0}^{x_{2}} \frac{\left(x_{2}-u\right)^{j}}{j !} \frac{u^{i_{2}}}{i_{2} !} \mathrm{d} u \\
& =\sum_{j=0}^{i_{1}} \frac{x_{1}^{i_{1}-j}}{\left(i_{1}-j\right) !} \frac{x_{2}^{j+i_{2}+1}}{\left(j+i_{2}+1\right) !} .
\end{aligned}
$$

By exploiting Lemma 1, expression (7) for $\hat{H}_{2}(x)$ can be rewritten to

$$
\begin{aligned}
\hat{H}_{2}(x) & =E_{k_{2}, \lambda}(x)-\sum_{i=0}^{k_{1}-1} \lambda^{i+k_{2}} e^{-\lambda\left(x+a_{1}\right)} \sum_{j=0}^{i} \frac{a_{1}^{i-j}}{(i-j) !} \frac{x^{j+k_{2}}}{\left(j+k_{2}\right) !} \\
& =E_{k_{2}, \lambda}(x)-\sum_{i=0}^{k_{1}-1} \sum_{j=0}^{i} \frac{\left(\lambda a_{1}\right)^{i-j}}{(i-j) !} e^{-\lambda a_{1}} \frac{(\lambda x)^{j+k_{2}}}{\left(j+k_{2}\right) !} e^{-\lambda x} \\
& =E_{k_{2}, \lambda}(x)-\sum_{i=k_{2}}^{k_{1}+k_{2}-1} \sum_{j=k_{2}}^{i} \frac{\left(\lambda a_{1}\right)^{i-j}}{(i-j) !} e^{-\lambda a_{1}} \frac{(\lambda x)^{j}}{j !} e^{-\lambda x}, \\
& x \geq 0 .
\end{aligned}
$$

The latter formula for $\hat{H}_{2}$, and also the formula for $\hat{H}_{1}$, can be generalized to an explicit, non-recursive formula for incomplete convolutions $\hat{H}_{r}$ with general $r \geq 1$.

In order to describe the generalization, we introduce sums of products of terms of the type $\left((\lambda x)^{j} / j !\right) e^{-\lambda x}$. For all $r \geq 1, x_{1}, \ldots, x_{r} \in R$ with $x_{1}, \ldots, x_{r} \geq 0, i_{2}, \ldots, i_{r} \in N_{0}$, and $i \in N_{0}$ with $i \geq \sum_{s=2}^{r} i_{s}$, we define

$$
\begin{aligned}
& P_{r}\left(x_{1}, \ldots, x_{r} ; i_{2}, \ldots, i_{r} ; i\right) \\
& :=\sum_{\left(j_{1}, \ldots, j_{r}\right) \in I_{r}\left(i_{2}, \ldots, i_{r} ; i\right)} \prod_{s=1}^{r} \frac{\left(\lambda x_{s}\right)^{j_{s}}}{j_{s} !} e^{-\lambda x_{s}},
\end{aligned}
$$

where the index set $I_{r}\left(i_{2}, \ldots, i_{r} ; i\right)$ is defined by

$$
\begin{aligned}
I_{r}\left(i_{2}, \ldots, i_{r} ; i\right):= & \left\{\left(j_{1}, \ldots, j_{r}\right) \in N_{0}{ }^{r} \mid \sum_{t=1}^{r} j_{t}=i,\right. \\
& \left.\sum_{t=s}^{r} j_{s} \geq \sum_{t=s}^{r} i_{s} \text { for } s=2, \ldots, r\right\} .
\end{aligned}
$$

Here, for $r=1$, the sequence $i_{2}, \ldots, i_{r}$ is empty, and we must read $I_{1}(; i)=\{(i)\}$ and

$P_{1}\left(x_{1} ; ; i\right)=\sum_{\left(j_{1}\right) \in I_{1}(; i)} \frac{\left(\lambda x_{1}\right)^{j_{1}}}{j_{1} !} e^{-\lambda x_{1}}=\frac{\left(\lambda x_{1}\right)^{i}}{i !} e^{-\lambda x_{1}}$.

Note that the sums $P_{r}\left(x_{1}, \ldots, x_{r} ; i_{2}, \ldots, i_{r} ; i\right)$ are always positive.

Theorem 1. For all $r \geq 1$,

$$
\begin{aligned}
& \hat{H}_{r}(x)=1-\sum_{s=1}^{r} \sum_{i=k_{r-s+2}+\ldots+k_{r}}^{k_{r-s+1}+\ldots+k_{r}-1} \\
& \quad P_{s}\left(a_{r-s+1}, \ldots, a_{r-1}, x ; k_{r-s+2}, \ldots, k_{r} ; i\right), x \geq 0
\end{aligned}
$$


(for $s=1$, read $k_{r-s+2}+\ldots+k_{r}=0$ and both sequences $a_{r-s+1}, \ldots, a_{r-1}$ and $k_{r-s+2}, \ldots, k_{r}$ are empty).

It is easily verified that the formula given by this theorem reduces to $\hat{H}_{1}(x)=E_{k_{1}, \lambda}(x)$ for $r=1$ and that it is equivalent to (8) for $r=2$. The proof of this theorem is rather technical and therefore it is postponed to the appendix.

The formula given by Theorem 1 shows that for all $r \geq 1$ and $x \geq 0, \hat{H}_{r}(x)$ is equal to 1 minus a finite sum of positive terms, where each term itself is a product of terms of the type $\left((\lambda x)^{j} / j !\right) e^{-\lambda x}$. Hence, using this formula will lead to a numerically stable formula for the evaluation of the incomplete convolutions $\hat{H}_{r}$. Finally, note that by Theorem 1 the following property is obtained: for $r \geq 2$,

$$
\begin{aligned}
\hat{H}_{r}(x)= & \left(\left(\ldots\left(E_{k_{2}, \lambda}\right)^{a_{2}} * \ldots * E_{k_{r-1}, \lambda}\right)^{a_{r-1}} * E_{k_{r}, \lambda}\right)(x) \\
& -\sum_{\substack{i=k_{2}+\ldots+k_{r} \\
k_{1}+\ldots+k_{r}-1}} P_{r}\left(a_{1}, \ldots, a_{r-1}, x ; k_{2}, \ldots, k_{r} ; i\right), \\
& x \geq 0 .
\end{aligned}
$$

By exploiting the results for the incomplete convolutions $\hat{H}_{r}$ itself, we easily obtain similar results for the integrals $\int_{a_{r}}^{\infty}\left(u-a_{r}\right) \mathrm{d} \hat{H}_{r}(u)$. For all $r \geq 1, x_{1}, \ldots, x_{r} \in \boldsymbol{R}$ with $x_{1}, \ldots, x_{r} \geq 0, i_{2}, \ldots, i_{r} \in N_{0}$, and $i \in N_{0}$ with $i \geq$ $\sum_{s=2}^{r} i_{s}$, define

$$
\begin{aligned}
& Q_{r}\left(x_{1}, \ldots, x_{r} ; i_{2}, \ldots, i_{r} ; i\right) \\
& :=\sum_{\left(j_{1}, \ldots, j_{r}\right) \in I_{r}\left(i_{2}, \ldots, i_{r} ; i\right)}\left(\prod_{s=1}^{r-1} \frac{\left(\lambda x_{s}\right)^{j_{s}}}{j_{s} !} e^{-\lambda x_{s}}\right) \\
& \quad \times \frac{1}{\lambda} \sum_{j=0}^{j_{r}} \frac{\left(\lambda x_{r}\right)^{j}}{j !} e^{-\lambda x_{r}} .
\end{aligned}
$$

Here, for $r=1$, read

$$
\begin{aligned}
Q_{1}\left(x_{1} ; ; i\right) & =\sum_{\left(j_{1}\right) \in I_{1}(; i)} \frac{1}{\lambda} \sum_{j=0}^{j_{1}} \frac{\left(\lambda x_{1}\right)^{j}}{j !} e^{-\lambda x_{1}} \\
& =\frac{1}{\lambda} \sum_{j=0}^{i} \frac{\left(\lambda x_{1}\right)^{j}}{j !} e^{-\lambda x_{\imath}} .
\end{aligned}
$$

Then we obtain the following theorem.

Theorem 2. For all $r \geq 1$,

$$
\begin{aligned}
& \int_{a_{r}}^{\infty}\left(u-a_{r}\right) \mathrm{d} \hat{H}_{r}(u) \\
& =\sum_{s=1}^{r} \sum_{i=k_{r-s+2}+\ldots+k_{r}}^{k_{r-s+1}+\ldots+k_{r}-1} Q_{s}\left(a_{r-s+1}, \ldots, a_{r} ; k_{r-s+2}, \ldots, k_{r} ; i\right)
\end{aligned}
$$

(for $s=1, k_{r-s+2}+\ldots+k_{r}=0$ and the sequence $k_{r-s+2}, \ldots$, $k_{r}$ is empty).

Proof. By using induction with respect to $i$, one can prove that for all $i \in N_{0}$ and $x \geq 0$,

$$
\int_{x}^{\infty}(u-x) \frac{\mathrm{d}}{\mathrm{d} u}\left\{-\frac{(\lambda u)^{i}}{i !} e^{-\lambda u}\right\} \mathrm{d} u=\frac{1}{\lambda} \sum_{j=0}^{i} \frac{(\lambda x)^{j}}{j !} e^{-\lambda x} .
$$

Next, by combining this result and Theorem 1, we find

$$
\begin{aligned}
& \int_{a_{r}}^{\infty}\left(u-a_{r}\right) \mathrm{d} \hat{H}_{r}(u)=\int_{a_{r}}^{\infty}\left(u-a_{r}\right) \frac{\mathrm{d}}{\mathrm{d} u}\left\{\hat{H}_{r}(u)\right\} \mathrm{d} u \\
& =\sum_{s=1}^{r} \sum_{i=k_{r-s+2}+\ldots+k_{r}}^{k_{r-s+1}+\ldots+k_{r}-1} \int_{a_{r}}^{\infty}\left(u-a_{r}\right) \\
& \times \frac{\mathrm{d}}{\mathrm{d} u}\left\{-P_{s}\left(a_{r-s+1}, \ldots, a_{r-1}, u ; k_{r-s+2}, \ldots, k_{r} ; i\right)\right\} \mathrm{d} u \\
& =\sum_{s=1}^{r} \sum_{i=k_{r-s+2}+\ldots+k_{r}}^{k_{r-s+1}+\ldots+k_{r}-1} \sum_{\left(j_{r-s+1}, \ldots, j_{r}\right) \in I_{s}\left(k_{r-s+2}, \ldots, k_{r} ; i\right)} \\
& \left(\prod_{t=r-s+1}^{r-1} \frac{\left(\lambda a_{t}\right)^{j_{t}}}{j_{t} !} e^{-\lambda a_{t}}\right) \\
& \times \int_{a_{r}}^{\infty}\left(u-a_{r}\right) \frac{\mathrm{d}}{\mathrm{d} u}\left\{-\frac{(\lambda u)^{j_{r}}}{j_{r} !} e^{-\lambda u}\right\} \mathrm{d} u \\
& =\sum_{s=1}^{r} \sum_{i=k_{r-s+2}+\ldots+k_{r}}^{k_{r-s+1}+\ldots+k_{r}-1} \sum_{\left(j_{r-s+1}, \ldots, j_{r}\right) \in I_{s}\left(k_{r-s+2}, \ldots, k_{r} ; i\right)} \\
& \left(\prod_{t=r-s+1}^{r-1} \frac{\left(\lambda a_{t}\right)^{j_{t}}}{j_{t} !} e^{-\lambda a_{t}}\right) \frac{1}{\lambda} \sum_{j=0}^{j_{r}} \frac{\left(\lambda a_{r}\right)^{j}}{j !} e^{-\lambda a_{r}} \\
& =\sum_{s=1}^{r} \sum_{i=k_{r-s+2}+\ldots+k_{r}}^{k_{r-s+1}+\ldots+k_{r}-1} Q_{s}\left(a_{r-s+1}, \ldots, a_{r} ; k_{r-s+2}, \ldots, k_{r} ; i\right) \text {. }
\end{aligned}
$$

Obviously, also the formula given by Theorem 2 is appropriate for numerical purposes. Further, by Theorem 2, we find that for all $r \geq 2$,

$$
\begin{aligned}
& \int_{a_{r}}^{\infty}\left(u-a_{r}\right) \mathrm{d} \hat{H}_{r}(u) \\
& =\int_{a_{r}}^{\infty}\left(u-a_{r}\right) \\
& \quad \times \mathrm{d}\left(\left(\ldots\left(E_{k_{2}, \lambda}\right)^{a_{2}} * \ldots * E_{k_{r-1}, \lambda}\right)^{a_{r-1}} * E_{k_{r}, \lambda}\right)(u) \\
& \quad+\sum_{i=k_{2}+\ldots+k_{r}}^{k_{1}+\ldots+k_{r}-1} Q_{r}\left(a_{1}, \ldots, a_{r} ; k_{2}, \ldots, k_{r} ; i\right) .
\end{aligned}
$$

\section{Numerical results for the multi-stage serial inventory system}

The explicit formulae derived in the previous section can be used for the two inventory systems described in Sect. 2, if the demand distribution $F$ is an Erlang distribution. As we will see below, those formulae can also be exploited if the demand distribution $F$ is a mixture of Erlang distributions with the same scale parameter. This is a useful property, since, by Schassberger [8], each continuous distribution $F$ on $[0, \infty)$ that is not equal to such a mixture, always can be approximated as accurately as desired by such a mixture. In particular, $F$ can be approximated by an $E_{k-1, k}$ distribution (i.e. a mixture of an Erlang- $(k-1)$ and an Erlang- $k$ distribution with the same scale parameter) with the same mean and standard deviation (or, equivalently, with the same first two moments) if $0<\sigma \leq \mu$, while in the other case, i.e. if $\sigma>\mu>0, F$ can be approximated by an $E_{1, k}$ (i.e. a mixture 
of an Erlang-1/exponential distribution and an Erlang- $k$ distribution with the same scale parameter) with the same mean and standard deviation. Explicit formulae for these fits are given by Tijms [10]. (Unfortunately, similar formulae are not available for more accurate fits on $F$.)

In this section, we focus on the multi-stage serial inventory system. Here, we assume that the demand distribution $F$ is equal to an $E_{k-1, k}$ distribution, i.e.

$F(x)=p E_{k-1, \lambda}(x)+(1-p) E_{k, \lambda}(x), \quad x \geq 0$,

where $k \geq 2, \lambda>0$ and $0<p \leq 1$. Under this assumption, the mean $\mu$ and standard deviation $\sigma$ of the demand per period are equal to $\mu=(k-p) / \lambda$ and $\sigma=\sqrt{k-p^{2}} / \lambda$. Both $\mu$ and $\sigma$ is positive. The coefficient of variation $c_{v a r}$ is equal to $c_{v a r}=\sqrt{k-p^{2}} /(k-p)$ and satisfies $c_{v a r} \leq 1$; here, an equality is obtained if and only if $k=2$ and $p=1$, i.e. if and only if $F$ is an exponential distribution. The other way round, if $\mu(>0)$ and $\sigma(0<\sigma \leq \mu)$ are given, then $k$ is such that $1 / k<c_{v a r}^{2} \leq 1 /(k-1)$ and

$p=\frac{1}{1+c_{v a r}^{2}}\left[k c_{v a r}^{2}-\sqrt{k\left(1+c_{v a r}^{2}\right)-k^{2} c_{v a r}^{2}}\right]$,

$\lambda=\frac{k-p}{\mu}$

(cf. Tijms [10]).

The assumption that the demand distribution $F$ is an $E_{k-1, k}$ distribution in fact means that in each period the demand is distributed according to an $E_{k-1, \lambda}$ distribution with probability $p$ and according to an $E_{k, \lambda}$ distribution with probability $1-p$. Hence, the total demand over $l(\geq 1)$ periods is distributed according to an $E_{(k-1) l+i, \lambda}$ distribution with probability $\left(\begin{array}{l}l \\ i\end{array}\right) p^{l-i}(1-p)^{i}, 0 \leq i \leq l$, and thus

$$
F_{l}(x)=\sum_{i=0}^{l}\left(\begin{array}{l}
l \\
i
\end{array}\right) p^{l-i}(1-p)^{i} E_{(k-1) l+i, \lambda}(x), \quad x \geq 0 .
$$

Similarly, for an $r$-fold incomplete convolution of convolutions of $F$, we find

$$
\begin{aligned}
\left(\left(\ldots\left(F_{k_{1}}\right)^{a_{1}} * \ldots * F_{k_{r-1}}\right)^{a_{r-1}} * F_{k_{r}}\right)(x) \\
=\sum_{0 \leq i_{1} \leq k_{1}, \ldots, 0 \leq i_{r} \leq k_{r}} \prod_{s=1}^{r}\left(\begin{array}{c}
k_{s} \\
i_{s}
\end{array}\right) p^{k_{s}-i_{s}}(1-p)^{i_{s}} \\
\quad\left(\left(\ldots\left(E_{(k-1) k_{1}+i_{1}, \lambda}\right)^{a_{1}} * \ldots * E_{(k-1) k_{r-1}+i_{r-1}, \lambda}\right)^{a_{r-1}}\right. \\
\left.\quad * E_{(k-1) k_{r}+i_{r}, \lambda}\right)(x), \quad x \geq 0,
\end{aligned}
$$

where $r \geq 1, a_{1}, \ldots, a_{r-1} \in \boldsymbol{R}$ with $a_{1}, \ldots, a_{r-1} \geq 0$, and $k_{1}, \ldots, k_{r} \in N$.

By combining (10) and the explicit formulae derived in the previous section, we can evaluate the incomplete convolutions occurring in the characterization of the optimal base stock policy $\left(S_{1}, \ldots, S_{N}\right)$ and the integrals occurring in formula (3) for the corresponding average costs $g\left(S_{1}, \ldots, S_{N}\right)$. We have implemented all these results in a Turbo Pascal program. In order to save computation time, special consideration has been given to the order in which the different terms (of the type $\left(\begin{array}{c}k_{s} \\ i_{s}\end{array}\right) p^{k_{s}-i_{s}}(1-p)^{i_{s}}$ and of the type $\left.\left((\lambda x)^{j} / j !\right) e^{-\lambda x}\right)$ are computed. For the same reason, the property stated in (9) has been exploited for the computation of the optimal base stock policy. To show the efficiency of
Table 1. The optimal base stock policy, the average costs and the corresponding computation times for the series of instances with varying values for the standard deviation $\sigma$ of the demand

\begin{tabular}{rrrrrrrrrrr}
\hline \multicolumn{4}{c}{$E_{k-1, k}$ distribution } & \multicolumn{2}{c}{ Optimal base stock policy } & \multicolumn{2}{c}{ Average costs } \\
$\sigma$ & $k$ & $p$ & $\lambda$ & $\left(S_{1}\right.$, & $S_{2}$, & $\left.S_{3}\right)$ & ctime & $g(\ldots)$ & ctime \\
\hline 10 & 101 & 1.0000 & 1.0000 & $(239$, & 549, & $747)$ & $25.3 \mathrm{~s}$ & 3246 & $2.9 \mathrm{~s}$ \\
20 & 26 & 1.0000 & 0.2500 & $(281$, & 600, & $794)$ & $1.7 \mathrm{~s}$ & 3819 & $0.2 \mathrm{~s}$ \\
30 & 12 & 0.6730 & 0.1133 & $(327$, & 654, & $843)$ & $0.4 \mathrm{~s}$ & 4417 & $0.1 \mathrm{~s}$ \\
40 & 7 & 0.5094 & 0.0649 & $(376$, & 709, & $892)$ & $0.2 \mathrm{~s}$ & 5037 & $0.1 \mathrm{~s}$ \\
50 & 6 & 1.0000 & 0.0400 & $(430$, & 767, & $943)$ & $0.1 \mathrm{~s}$ & 5690 & $0.0 \mathrm{~s}$ \\
60 & 3 & 0.1202 & 0.0288 & $(485$, & 825, & $993)$ & $0.1 \mathrm{~s}$ & 6347 & $0.0 \mathrm{~s}$ \\
70 & 3 & 0.8222 & 0.0218 & $(546$, & 887, & $1045)$ & $0.1 \mathrm{~s}$ & 7047 & $0.0 \mathrm{~s}$ \\
80 & 2 & 0.2631 & 0.0174 & $(602$, & 946, & $1096)$ & $0.1 \mathrm{~s}$ & 7713 & $0.0 \mathrm{~s}$ \\
90 & 2 & 0.5545 & 0.0145 & $(666$, & 1009, & $1149)$ & $0.1 \mathrm{~s}$ & 8434 & $0.0 \mathrm{~s}$ \\
100 & 2 & 1.0000 & 0.0100 & $(749$, & 1081, & $1204)$ & $0.0 \mathrm{~s}$ & 9269 & $0.0 \mathrm{~s}$ \\
\hline
\end{tabular}

the program, we present some numerical results and the corresponding computation times which were needed to obtain them.

We consider two series of instances. In the first series, we take

$N=3, l_{1}=1, l_{2}=3, l_{3}=2, \mu=100$,

$h_{1}=1, h_{2}=3, h_{3}=6, p=200$,

and we vary the standard deviation $\sigma$ of the demand. The obtained results have been presented in Table 1. In the first four columns of this table, we have listed the values of $\sigma$ and the corresponding values for the parameters of the $E_{k-1, k}$ distribution of the demand. In the other columns, the optimal base stock policy $\left(S_{1}, S_{2}, S_{3}\right)$, the average costs $g\left(S_{1}, S_{2}, S_{3}\right)$, and the required computation times are given. The denoted computation times are in seconds and have been obtained on a PC 486 AT/DX2, $66 \mathrm{MHz}$.

The results show that the computation times for both the optimal base stock policy and the corresponding average costs are small. A somewhat larger computation time has been obtained for the optimal base stock policy for the case $\sigma=10$, which is due to the fact that the $E_{k-1, k}$ distribution of the demand consists of many phases in that case. Further, we see that both the order-up-to levels and the minimal average costs are monotonously decreasing for decreasing values of $\sigma$ (for $\sigma \downarrow 0,\left(S_{1}, S_{2}, S_{3}\right)$ and $g\left(S_{1}, S_{2}, S_{3}\right)$ decrease monotonously to $\left(l_{1}+1, l_{2}+l_{1}+1, l_{3}+l_{2}+l_{1}+1\right) \mu=$ $(200,500,700)$ and the pipeline costs $h_{3} l_{2}+\left(h_{3}+h_{2}\right) l_{2}=$ 2700 , respectively).

In the second series of instances, we take

$N=10, l_{n}=1$ for $n=1, \ldots, 10, \mu=100, \sigma=40$, $h_{n}=1$ for $n=1, \ldots, 10, p=200$,

and we vary the number of installations that are allowed to hold stock. We assume that the installations $1, \ldots, \tilde{N}$ are allowed to hold (safety) stock and that the installations $\tilde{N}+$ $1, \ldots, N$ are forced to be stockless inventory points (this is obtained by giving each of these installations the same order-up-to level as installation $\tilde{N}$ ). The value of $\tilde{N}$ is varied from 1 to 10 . For each $\tilde{N}$, the optimal base stock policy $\left(S_{1}, \ldots, S_{10}\right)$ is given by $S_{n}=\tilde{S}_{n}$ for $n=1, \ldots, \tilde{N}$ and $S_{n}=$ $\tilde{S}_{\tilde{N}}$ for $n=\tilde{N}+1, \ldots, 10$, where $\tilde{S}_{1}, \ldots, \tilde{S}_{\tilde{N}}$ are the orderup-to levels of the optimal base stock policy $\left(\tilde{S}_{1}, \ldots, \tilde{S}_{\tilde{N}}\right)$ of the $\tilde{N}$-stage serial inventory system with the same demand distribution and penalty costs as for the $N$-stage system and with the following leadtimes and inventory holding costs: 
Table 2. The optimal base stock policy, the average costs and the corresponding computation times for the series of instances with a varying number of stockholding installations $\tilde{N}$

$\tilde{N}$ Optimal base stock policy Average costs

$\tilde{N}\left(S_{1}, \quad S_{2}, S_{3}, S_{4}, \quad S_{5}, \quad S_{6}, \quad S_{7}, S_{8}, \quad S_{9}, S_{10}\right)$ ctime $g(\ldots)$ ctime

$1(1330,1330,1330,1330,1330,1330,1330,1330,1330,1330) 0.0 \mathrm{~s} 74430.0 \mathrm{~s}$

$2(376,1335,1335,1335,1335,1335,1335,1335,1335,1335) 0.6 \mathrm{~s} 73780.1 \mathrm{~s}$

$3(376,495,1339,1339,1339,1339,1339,1339,1339,1339) 1.0 \mathrm{~s} 73230.1 \mathrm{~s}$

$4(376,495,612,1344,1344,1344,1344,1344,1344,1344) 1.5 \mathrm{~s} 72780.2 \mathrm{~s}$

$5(376,495,612,726,1349,1349,1349,1349,1349,1349) 2.1 \mathrm{~s} 72400.4 \mathrm{~s}$

$6(376,495,612,726,838,1354,1354,1354,1354,1354) 3.1 \mathrm{~s} 72100.6 \mathrm{~s}$

$7(376,495,612,726,838,949,1359,1359,1359,1359) 4.2 \mathrm{~s} 71860.9 \mathrm{~s}$

$8(376,495,612,726,838,949,1058,1365,1365,1365) 5.2 \mathrm{~s} 71681.3 \mathrm{~s}$

$9(376,495,612,726,838,949,1058,1166,1372,1372) 5.9 \mathrm{~s} 71541.7 \mathrm{~s}$

$10(376,495,612,726,838,949,1058,1166,1273,1380) 6.2 \mathrm{~s} 71471.9 \mathrm{~s}$

$\tilde{l}_{n}=l_{n}$ for $n=1, \ldots, \tilde{N}-1, \quad \tilde{l}_{\tilde{N}}=11-\tilde{N}$,

$\tilde{h}_{n}=h_{n}$ for $n=1, \ldots, \tilde{N}-1, \quad \tilde{h}_{\tilde{N}}=11-\tilde{N}$.

In this $\tilde{N}$-stage system, the stages in front of the installations $N, \ldots, \tilde{N}+1, \tilde{N}$ are seen as one large stage. The average costs $g\left(S_{1}, \ldots, S_{10}\right)$ are obtained by computing the average costs $\tilde{g}\left(\tilde{S}_{1}, \ldots, \tilde{S}_{\tilde{N}}\right)$ of the optimal base stock policy $\left(\tilde{S}_{1}, \ldots, \tilde{S}_{\tilde{N}}\right)$ for the $\tilde{N}$-stage system and next adding $\sum_{n=\tilde{N}}^{N-1}\left(\sum_{i=n}^{N} h_{i}\right) \mu$ to correct for the pipeline costs in the stages in front of the installations $N-1, \ldots, \tilde{N}$ (these costs are neglected in the $\tilde{N}$-stage system). Alternatively, one can compute them directly by substituting $\left(y_{1}, \ldots, y_{10}\right)=$ $\left(S_{1}, \ldots, S_{10}\right)$ into formula (3). The results for this second series of instances are listed in Table 2.

Again it may be concluded that the computation times are small. A remarkable result is that the computation times increase only linearly for increasing values of the number of stockholding installations $\tilde{N}$. The last but one column in the table shows the decrease in costs when more installations are allowed to hold stock. In the situation where all installations are allowed to hold stock (i.e. for $\tilde{N}=10$ ) the costs are $4.0 \%$ lower than in the situation where only the most downstream installation is allowed to hold stock (i.e. for $\tilde{N}=1$. This percentage is not very high, but it increases to $10.1 \%$ if we subtract the pipeline costs $(=4500)$ in both situations. Finally, we see that a large part of these savings of $10.1 \%$ is already obtained when only a few intermediate installations are allowed to hold stock.

\section{Epilogue}

We have shown the relevance of incomplete convolutions for (multi-stage) production and inventory systems. For the case where the underlying distributions are (mixtures of) Erlang distributions with the same scale parameter, we have derived explicit formulae that are appropriate for the numerical evaluation of incomplete convolutions. These formulae are useful for the two inventory systems that we have described. However, for other systems, among which the multi-stage serial production system, they can not be used in general. Hence, it is desired to have also such explicit formulae available for other cases, for example for gamma distributions and for Erlang distributions with possibly different scale parameters.
As an alternative for the exact approach as promoted above, one can use the approximate method that has been presented in [12]. This method works as follows. Given the first two moments of a random variable $X_{1}$, a mixture of Erlang distributions is fitted on $X_{1}$. Here, an $E_{k-1, k}$ distribution as described in Sect. 4 is used if $X_{1}$ has a coefficient of variation that is smaller than or equal to 1 , and an hyperexponential distribution is used otherwise (note that this hyperexponential distribution is a mixture of Erlang distributions with possibly different scale parameters and therefore it can not be used for exact computations as presented in Sect. 4). Next, we are able to derive an expression for the distribution of $\left(X_{1}-a_{1}\right)^{+}$and hence for the first two moments of $\left(X_{1}-a_{1}\right)^{+}$. If a second random variable $X_{2}$ is given that is independent of $X_{1}$, then we immediately find the first two moments of $\left(X_{1}-a_{1}\right)^{+}+X_{2}$. A repeated application of this procedure yields the first two moments and distributions of the general multiple incomplete convolutions $\hat{G}_{r}$, as described in the introduction. So, this method also can be used for the multi-stage serial production system. In [12], this method has been applied to the multi-stage serial inventory system and it has been shown that in general the order-up-to levels of the optimal base stock policy and the corresponding average costs generated by the approximate method are within $2.0 \%$ of the exact results obtained by the procedures described in this paper.

Another approximate method for evaluating incomplete convolutions has been described by Badinelli [1]. His method is based on orthogonal polynomials and also requires only a small amount of computational effort. The accuracy of this method strongly depends on the type of orthogonal polynomials that is used and the choices of computational factors. Hence, the method seems more complicated to implement. It is not known what magnitude of errors can be expected when this method is used to solve the multi-stage serial inventory system, for example.

Finally, it is noted that in fact we have studied one specific type of incomplete convolutions. Another type of incomplete convolutions arises in the analysis of the uncapacitated single-stage inventory system with lost sales. It appears that, for the case where the underlying distributions are Erlang distributions with the same scale parameter, similar explicit formulae can be obtained as for the type that has been studied in this paper; see Rutten et al. [7] (see also [11]). Hence, also this lost sales system can be efficiently solved/evaluated by exploiting these formulae.

\section{Appendix. Proof of Theorem 1}

For the proof of Theorem 1, we need the following preliminary result.

Lemma 2. For all $r \geq 1, x_{1}, \ldots, x_{r} \in \boldsymbol{R}$ with $x_{1}, \ldots, x_{r} \geq$ $0, x \in \boldsymbol{R}$ with $x \geq 0, i_{2}, \ldots, i_{r} \in N_{0}, i \in N_{0}$ with $i \geq$ $\sum_{s=2}^{r} i_{s}$, and $k \in \bar{N}$,

$$
\begin{aligned}
& \int_{0}^{x} P_{r}\left(x_{1}, \ldots, x_{r-1}, x+x_{r}-u ; i_{2}, \ldots, i_{r} ; i\right) \mathrm{d} E_{k, \lambda}(u) \\
& =P_{r+1}\left(x_{1}, \ldots, x_{r}, x ; i_{2}, \ldots, i_{r}, k ; i+k\right) .
\end{aligned}
$$


Proof. With the convention that $\prod_{s=1}^{r-1}(\ldots)=1$ for $r=1$, we find that for all $r \geq 1$,

$$
\begin{aligned}
& \int_{0}^{x} P_{r}\left(x_{1}, \ldots, x_{r-1}, x+x_{r}-u ; i_{2}, \ldots, i_{r} ; i\right) \mathrm{d} E_{k, \lambda}(u) \\
& =\int_{0}^{x} \sum_{\left(j_{1}, \ldots, j_{r}\right) \in I_{r}\left(i_{2}, \ldots, i_{r} ; i\right)}\left(\prod_{s=1}^{r-1} \frac{\left(\lambda x_{s}\right)^{j_{s}}}{j_{s} !} e^{-\lambda x_{s}}\right) \\
& \quad \times \frac{\left(\lambda\left(x+x_{r}-u\right)\right)^{j_{r}}}{j_{r} !} e^{-\lambda\left(x+x_{r}-u\right)} \mathrm{d} E_{k, \lambda}(u) \\
& =\sum_{\left(j_{1}, \ldots, j_{r}\right) \in I_{r}\left(i_{2}, \ldots, i_{r} ; i\right)}\left(\prod_{s=1}^{r-1} \frac{\left(\lambda x_{s}\right)^{j_{s}}}{j_{s} !} e^{-\lambda x_{s}}\right) \\
& \quad \times \int_{0}^{x} \frac{\left(\lambda\left(x+x_{r}-u\right)\right)^{j_{r}}}{j_{r} !} e^{-\lambda\left(x+x_{r}-u\right)} \mathrm{d} E_{k, \lambda}(u) .
\end{aligned}
$$

By using Lemma 1, for the integral in this formula, we find

$$
\begin{aligned}
& \int_{0}^{x} \frac{\left(\lambda\left(x+x_{r}-u\right)\right)^{j_{r}}}{j_{r} !} e^{-\lambda\left(x+x_{r}-u\right)} \mathrm{d} E_{k, \lambda}(u) \\
& =\lambda^{j_{r}+k} e^{-\lambda\left(x+x_{r}\right)} \int_{0}^{x} \frac{\left(x+x_{r}-u\right)^{j_{r}}}{j_{r} !} \frac{u^{k-1}}{(k-1) !} \mathrm{d} u \\
& =\lambda^{j_{r}+k} e^{-\lambda\left(x+x_{r}\right)} \sum_{j=0}^{j_{r}} \frac{x_{r}^{j_{r}-j}}{\left(j_{r}-j\right) !} \frac{x^{j+k}}{(j+k) !} \\
& =\sum_{j=0}^{j_{r}} \frac{\left(\lambda x_{r}\right)^{j_{r}-j}}{\left(j_{r}-j\right) !} e^{-\lambda x_{r}} \frac{(\lambda x)^{j+k}}{(j+k) !} e^{-\lambda x},
\end{aligned}
$$

and hence we can continue with

$$
\begin{aligned}
& \int_{0}^{x} P_{r}\left(x_{1}, \ldots, x_{r-1}, x+x_{r}-u ; i_{2}, \ldots, i_{r} ; i\right) d E_{k, \lambda}(u) \\
& =\sum_{\left(j_{1}, \ldots, j_{r}\right) \in I_{r}\left(i_{2}, \ldots, i_{r} ; i\right)}\left(\prod_{s=1}^{r-1} \frac{\left(\lambda x_{s}\right)^{j_{s}}}{j_{s} !} e^{-\lambda x_{s}}\right) \\
& \sum_{j=0}^{j_{r}} \frac{\left(\lambda x_{r}\right)^{j_{r}-j}}{\left(j_{r}-j\right) !} e^{-\lambda x_{r}} \frac{(\lambda x)^{j+k}}{(j+k) !} e^{-\lambda x} \\
& =\sum_{\left(j_{1}, \ldots, j_{r}\right) \in I_{r}\left(i_{2}, \ldots, i_{r} ; i\right)}\left(\prod_{s=1}^{r-1} \frac{\left(\lambda x_{s}\right)^{j_{s}}}{j_{s} !} e^{-\lambda x_{s}}\right) \\
& \sum_{\substack{j, j^{\prime} \in\{0,1, \ldots\} \\
j+j^{\prime} \leq j_{r}+k, j^{\prime} \geq k}} \frac{\left(\lambda x_{r}\right)^{j}}{j !} e^{-\lambda x_{r}} \frac{(\lambda x)^{j^{\prime}}}{j^{\prime} !} e^{-\lambda x} \\
& =\sum_{\left(j_{1}, \ldots, j_{r-1}, j, j^{\prime}\right) \in I_{r+1}\left(i_{2}, \ldots, i_{r}, k, i+k\right)}\left(\prod_{s=1}^{r-1} \frac{\left(\lambda x_{s}\right)^{j_{s}}}{j_{s} !} e^{-\lambda x_{s}}\right) \\
& \frac{\left(\lambda x_{r}\right)^{j}}{j !} e^{-\lambda x_{r}} \frac{(\lambda x)^{j^{\prime}}}{j^{\prime} !} e^{-\lambda x} \\
& =P_{r+1}\left(x_{1}, \ldots, x_{r}, x ; i_{2}, \ldots, i_{r}, k ; i+k\right) \text {. }
\end{aligned}
$$

Proof of Theorem 1. The proof is given by induction with respect to $r$. For $r=1$, the formula given in Theorem 1 reduces to $\hat{H}_{1}(x)=E_{k_{1}, \lambda}(x), x \geq 0$, and hence is correct. Now suppose that the formula holds for some $r \geq 1$. Then, for $r+1$, we find

$$
\begin{aligned}
\hat{H}_{r+1}(x)= & \left(\hat{H}_{r}^{a_{r}} * E_{k_{r+1}, \lambda}\right)(x) \\
= & \int_{0}^{x} \hat{H}_{r}\left(x+a_{r}-u\right) \mathrm{d} E_{k_{r+1}, \lambda}(x) \\
= & \int_{0}^{x}\left(1-\sum_{s=1}^{r} \sum_{i=k_{r-s+2}+\ldots+k_{r}}^{k_{r-s+1+\ldots+k_{r}-1}}\right. \\
& \left.P_{s}\left(a_{r-s+1}, \ldots, a_{r-1}, x+a_{r}-u ; k_{r-s+2}, \ldots, k_{r} ; i\right)\right) \\
& \times \mathrm{d} E_{k_{r+1}, \lambda}(x) \\
= & E_{k_{r+1}, \lambda}(x)-\sum_{s=1}^{r} \sum_{i=k_{r-s+2}+\ldots+k_{r}}^{k_{r-s+1}+\ldots+k_{r}-1} \\
& \int_{0}^{x} P_{s}\left(a_{r-s+1}, \ldots, a_{r-1}, x+a_{r}-u ; k_{r-s+2}, \ldots, k_{r} ; i\right) \\
& \times \mathrm{d} E_{k_{r+1}, \lambda}(x), x \geq 0 .
\end{aligned}
$$

Here, we apply Lemma 2 , and we continue with

$$
\begin{aligned}
\hat{H}_{r+1}(x) & =E_{k_{r+1}, \lambda}(x)-\sum_{s=1}^{r} \sum_{i=k_{r-s+2}+\ldots+k_{r}}^{k_{r-s+1+\ldots+k_{r}}-1} \\
& P_{s+1}\left(a_{r-s+1}, \ldots, a_{r}, x ; k_{r-s+2}, \ldots, k_{r}, k_{r+1} ; i+k_{r+1}\right) \\
& =E_{k_{r+1}, \lambda}(x)-\sum_{s=1}^{r} \sum_{i=k_{r-s+2}+\ldots+k_{r+1}}^{k_{r-s+1}+\ldots+k_{r+1}-1} \\
& P_{s+1}\left(a_{r-s+1}, \ldots, a_{r}, x ; k_{r-s+2}, \ldots, k_{r+1} ; i\right) \\
& =E_{k_{r+1}, \lambda}(x)-\sum_{s=2}^{r+1} \sum_{i=k_{r-s+3}+\ldots+k_{r+1}}^{k_{r-s+2+\ldots+k_{r+1}-1}} \\
& P_{s}\left(a_{r-s+2}, \ldots, a_{r}, x ; k_{r-s+3}, \ldots, k_{r+1} ; i\right) \\
& =1-\sum_{s=1}^{r+1} \sum_{i=k_{r-s+3}+\ldots+k_{r+1}}^{k_{r-s+2}+\ldots+k_{r+1}-1} \\
& P_{s}\left(a_{r-s+2}, \ldots, a_{r}, x ; k_{r-s+3}, \ldots, k_{r+1} ; i\right),
\end{aligned}
$$

which shows that the formula given in Theorem 1 also holds for $r+1$. This completes the proof.

\section{References}

1. Badinelli RD (1996) Approximating probability density functions and their convolutions using orthogonal polynomials. Eur I Oper Res 95:211-230

2. Clark AJ, Scarf H (1960) Optimal policies for a multi-echelon inventory problem. Manag Sci 6:475 490

3. Federgruen A, Zipkin PH (1986) An inventory model with limited production capacity and uncertain demands, I. The average cost criterion. Math Oper Res 11:193-207

4. Gerchak Y, Gupta D, Henig M (1997) Reservation planning for elective surgery under uncertain demand for emergency surgery. Manag Sci (to appear)

5. Gong L, De Kok AG, Ding J (1994) Optimal leadtimes planning in a serial production system. Manag Sci 40:629-632

6. Langenhoff LJG, Zijm WHM (1990) An analytical theory of multiechelon production/distribution systems. Stat Neerl 44:149-174

7. Rutten WGMM, Van Donselaar K, De Kok AG, Regterschot GJK (1992) The lost sales inventory model. Research report TUE/BDK/ LBS/92-04, Graduate School of Industrial Engineering and Management Science, Endhoven University of Technology

8. Schassberger R (1973) Wanteschlangen. Springer-Verlag, Berlin 
9. Tayur SR (1993) Computing the optimal policy for capacitated inventory models. Commun Stat-Stoch Mod 9:585-598

10. Tijms HC (1994) Stochastic Models: An Algorithmic Approach. Wiley, New York

11. Van Donselaar K, De Kok AG, Rutten WGMM (1996) Two replenishment strategies for the lost sales inventory model: A comparison. Int $\mathrm{J}$ Prod Econ 46:297-310

12. Van Houtum GJ, Zijm WHM (1991) Computational procedures for stochastic multi-echelon production systems. Int J Prod Econ 23:223237
13. Van Houtum GJ, Inderfurth K, Zijm WHM (1996) Materials coordination in stochastic multi-echelon systems. Eur J Oper Res 95:1-23

14. Yano CA (1987) Setting planned leadtimes in serial production systems with tardiness costs. Manag Sci 33:95-106

15. Zijm WHM, Van Houtum GJ (1994) On multi-stage production/inventory systems under stochastic demand. Int $J$ Prod Econ $35: 391-400$ 